der Ärzte, die sie als Nebenfach betrieben, bis sie schliesslich selbständig wurde; und ebenso kann die Technologie heute nicht mehr lediglich als ein Nebenfach der reinen Chemie betrachtet und von solchen gelehrt werden, die nicht wenigstens einige Zeit lang in Fabrikbetrieben thätig gewesen sind. Niemand wird ein Technologe, indem er allein aus Büchern und Mittheilungen Anderer lernt, ebenso wie auch niemand ein Künstler wird, indem er Kunstgeschichte treibt und Museen besucht; eigene praktische Thätigkeit ist in beiden Berufen unumgänglich nothwendig. So muss denn auch der Lehrer der chemischen Technologie selbst in der Lage gewesen sein, die sich bei der Ausübung seines Faches ergebenden Schwierigkeiten aus eigener Anschauung kennen zu lernen. Auch wird er nur durch Erfahrung die Grundlagen gewinnen, nach denen er die Angaben ron Erfindern und Fabrikanten beurtheilen kann.

Es ist ferner keine Gefahr vorhanden, dass der künftige Lehrer durch die Thätigkeit in einem Fabrikbetriebe von seinem theoretischen Wissen so viel einbüsst, dass er nun für die Vertretung einer Wissenschaft unbrauchbar würde; denn es wird auch in unseren Fabriken wissenschaftlich genug gearbeitet, nicht nur in den Versuchs- und analytischen Laboratorien, sondern auch im Betriebe selbst. Es wird ihm in letzterem reichlicher Anlass geboten, wissenschaftliche Fragen aufzuwerfen und zu beantworten, da hier durchaus nicht alles so abläuft, wie in der Theorie oder im Laboratorium und deshalb überall zu untersuchen ist, warum dies so ist. Aus dem nämlichen Grunde wäre es übrigens nicht nur für den Technologen, sondern auch für den Analytiker wünschenswerth, erst einige Zeit in einer Fabrik gearbeitet zu haben, wo oft Bestandtheile ihrer Menge nach zu bestimmen sind, die man im Unterrichtslaboratorium einfach als Spuren bezeichnet, und wo man nicht nur genau arbeiten lernt, sondern auch schnell, ein Erforderniss, auf das in unseren Hochschulen viel zu wenig Werth gelegt wird.

Ein weiterer Einwand gegen die Besetzung der neuen Lehrstühle mit nicht nur theoretisch vorgebildeten Fachgenossen ist der, dass sich für die verhältnissmässig geringen hierfür ausgesetzten Gehälter wohl keine tüchtigen Leute aus der Praxis finden würden. Dieser Einwand ist wohl nur eine blosse Annahme; wenn erst die Lehrstühle geschaffen sind, wird an geeigneten Personen kein Mangel sein. Denn das Lehramt ist ein Beruf, der um seinetwillen ausgeübt wird, und nicht allein um des Geldes willen. Freilich muss das damit verbundene Gebalt so hoch sein, dass der Lehrer auch davon leben kann und nicht noch auf Privatvermögen angewiesen ist. Ebenso müssten auch die Gehälter der Assistenten an den Unterrichtslaboratorien etwas höher sein, als sie jetzt sind, sodass man nicht mehr genöthigt wäre, diese wichtigen Posten mit jungen Leuten zu besetzen, die eben ausstudirt haben oder gar selbst noch studiren, und die, da es ihnen somit an Tiefe des Wissens nothwendig mangelt, nicht die Fähigkeit haben, zu unterrichten. Vielfach haben sie auch garnicht die Zeit dazu, da sie mit eigenen Arbeiten $\mathrm{zu}$ sehr beschäftigt sind, sodass sie thatsächlich eher Stipendieninhaber sind als Lehrer.
Es sollten auch diese Lehrstellen nur mit solchen besetzt werden, die einige Jahre Praxis hinter sich haben, und die nicht nur in den Laboratorien zugegen, sondern auch von Nutzen sein können. Selbstverständlich müssten sie dann Aussicht haben, nach nicht zu langer Zeit in die eigentlichen Lehrstellen aufzurücken, sodass damit auch der häufige Wechsel der Assistenten aufhören würde, der jetzt in grossen Laboratorien die Erfolge des Unterrichts so sehr behindert. Der Zweck der Unterrichtslaboratorien ist einzig und allein der Unterricht, und diesem $Z$ wecke hat sich alles an ihnen anzupassen.

\section{Die Verwerthung der Sulfitstoffablange.}

Unter diesem Titel hatte Herr Dr. Harp die Freundlichkeit, über meinen Vortrag auf dem III. internationalen Congress für angewandte Chemie in Wien in dieser Zeitschrift zu referiren. Da ibm dabei manche Ungenauigkeiten untergelaufen sind, sei es mir gestattet, die auffallendsten derselben wie folgt richtigzustellen :

Ich habe nie daran gedacht, dass man die Verbindung von Furfurol mit Anilin als Farbstoff verwenden könne, sondern ausdrücklich das Gegentheil gesagt (S. 876, Z. 10. v. u.).

S. 877, Z. 27 v. o. soll es heissen statt „Carbonylverbindung" richtig: "Carbonylgruppe".

S. 877 Z. 33 v. o. statt in schwedischen Zeitschriften" ricbtig: „von dem schwedischen Chemiker Klason".

Zu der Angabe S. 877 S. 22 v. u. muss ich bemerken, dass ich nicht behauptet habe, Lindsey habe von Ligninsulfosäure gesprochen, sondern lediglich gesagt, dass die Formel meiner freien Säure mit der Formel der von Lindsey untersuchten ,organischen Substanz" übereinstimmt.

S. $878 \mathrm{Z} .17$ v. u. soll es statt „Dornfeld “ richtig heissen "Dorenfeldt".

S. 879 Z. 3 v. o. u. 20 v. o. soll es statt Beutner \& Drewsen richtig heissen: „Dr. Viggs Beutner Drewsen in Bousdalen".

S. 880 Z. 14 v. u. soll es statt "Safranol" heissen: "Safranin".

S. 880 Z. 22 v. o. soll es statt Dinitrophenylpropiolsäure" heissen "Nitrophenylpropiolsäure".

S. 881 Z. 7. v. u.: Bei meinem Beizprocess mittels Liguorosin wird nicht 40 Proc. des ,färbenden Materials" erspart, sondern die Ausnutzung des Chroms ist eine vollständige, während bei der Anwendung ron Weinstein nur 60 Proc. ausgenutzt werden.

Wien, k. k. Techn. Gew.-Museum.

$$
\text { Ir. Heinrich Seidel. }
$$

\section{Seidenglanz auf Banmwolle.}

Im 43. Heft dieser Zeitschrift streift Dr. A. Buntrock in seinem "Bericht über die Neuerungen auf dem Gebiete der Veredelung der Gespinnstfasern" zweimal, und zwar auf S. 987 und 990, 
das aus der Färberei Glauchau rorm. Otto Seyfert in Glauchau hervorgegangene, in Frankreich unter No. 262471 patentirte Verfahren: „Baumwolle unter Erzeugung von Seidenglanz und Vermeidung des Einschrumpfens zu mercerisiren."

Dieser Bericht enthält insofern eine Entstellung des dem genannten französischen Patent No. 262471 zu Grunde liegenden Patentanspruches, indem darin, $d . b$. in ersterem gesagt wird, dass die Spannung bez. Streckung der mit Alkalien getränkten Baumwolle während des Trocknens vorgenommen werde, was aber nicht stimmt, und lassen wir zur Klarstellung dieses Punktes den betreffenden Patentanspruch hier sowohl im französischen Urtheil, als auch in der deutschen Übersetzung folgen.

„Un procédé pour donner au coton l'aspect luisant de la soie, procédé qui consiste à traiter les fibres végétales sous forme d'échevaux ou de tissus par des alcalis caustiques et à les sécher, fortement tendues, à une température modérée.

„Ein Process, der Baumwolle das glänzende Aussehen von Seide zu geben, welcher daraus besteht, die vegetabilischen Fasern in Strangoder Gewebeform mit kaustischen Alkalien zu behandeln, und sie, stark gespannt, in einer mässigen Temperatur zu trocknen."

Wenn der Herr Berichterstatter auf Grund seiner unrichtigen Wiedergabe obigen Patentanspruches zu dem Schlusse kommt, dass dieses Verfahren, also das von ihm falsch beschriebene (mit Spannung während des Trocknens) technisch schwer durchzuführen sein dürfte, so trifft das für dieses zu, trifft aber nicht $z u$ für das unsrige, bei welchem in gespanntem Zustande getrocknet, also erst gespannt und dann getrocknet (unter Beibehaltung der Spannung) wird.

Damit wird auch die in dem Bericht des Herrn Dr. A. Buntrock aufgestellte Behauptung, unser Verfahren charakterisire sich als ein sogenanntes Umgehungspatent, bez. eine Nachahmung des Thomas \& Prevost'schen widerlegt. Denn abgesehen davon, dass unsere Versuche und $\mathrm{Pa}$ tentanmeldungen aus derselben Zeit herrühren wie die Thomas \& Prevost'schen und also keine Nachahmung dieser sein können, beruht unser Verfahren auf der Fixirung der durch Natronlauge angegriffenen Substanz mittels Trocknens, d. h. die mercerisirte, g edehnte Faser wird bei gelinder Temperaturerhöhung so lange der Wärme- und Wasserentziehung ausgesetzt, bis sie ihr Einschrumpfungsbestreben verloren hat, und hierauf das Natron gewöhnlich, also ohne Spannung, ausgewaschen.

Principiell ganz anders verläuft die Fixirung der durch Natronlauge eingeschrumpften Cellulose bei Thomas \& Prevost, indem da das Wasser während des Waschens in gestrecktem Zustande die Contractionstendenz der Faseroberfläche unter Entfernung des Natrons beseitigt.

Auf die technisch leichtere Handhabung des unsrigen Verfahrens einzugehen, würde zu weit führen, aber schon im Mittel der Fixirung (und rermuthlich auch im chemischen oder physikalischen
Vorgange hierbei) unterscheidet es sich völlig von dem der Firma Thomas \& Prevost sowohl als von dem von deren Vorgänger Lowe.

$$
\text { Fürberei Glauchau vorm. Otto Seyfert. }
$$

\section{Elektrochemie.}

Darstellung von m-Diamidohydrazobenzol. Nach Anilinölfabrik A. Wülfing (D.R.P. No. 100 233) gelingt es durch elektrolytische Reduction leicht, aus $\mathrm{m}-\mathrm{Ni}-$ tranilin 90 Proc. der theoretischen Ausbeute an $m$-Diamidoazobenzol zu erhalten und diese Verbindung weiter zu m-Diamidohydrazobenzol zu reduciren mit über 80 Proc. der berechneten Ausbeute. Die Umlagerung dieses Productes in $m$-Diamidobenzidin vom Schmelzpunkt $166^{\circ}$ geht auf die bekannte Weise leicht vor sich.

Die elektrolytische Reduction des $\mathrm{m}-\mathrm{Ni}$ tranilins zu m-Diamidoazobenzol bez. m-Diamidohydrazobenzol lässt sich unter verschiedenen Versuchsbedingungen erzielen. Beispielsweise löst man $1 \mathrm{k} \mathrm{m}$-Nitranilin und $1 / 4 k$ Natriumacetat (vergl. Pat. 100 234) in $8 \mathrm{k}$ Weingeist von etwa 50 bis 60 Proc. und behandelt diese Lösung in der Kathodenkammer zuerst mit 770 Ampèrestunden bei einer Stromdichte von 1000 bis 2000 Ampère auf $1 \mathrm{qm}$. Es erfolgt Umwandlung in m-Diamidoazobenzol. Behandelt man dieses hierauf mit weiteren 200 bis 220 Ampèrestunden bei einer Stromdichte von 200 bis $100 \mathrm{Amp}$. auf $1 \mathrm{qm}$, so erhält man m-Diamidohydrazobenzol.

Handelt es sich um die Gewinnung des m-Diamidoazobenzols, so lässt man die Kathodenflüssigkeit nach Zufuhr von 770 Ampèrestunden erkalten, wobei fast die Gesammtmenge des Azokörpers in orangefarbigen Nadeln auskrystallisirt. Bei der Darstellung des $m$-Diamidohydrazobenzols fällt im Verlauf der weiteren Stromzufuhr der sehr schwer lösliche Hydrazokörper als sandiges Krystallpulver nahezu vollkommen aus. Das Hydrazoanilin krystallisirt in farblosen, stark lichtbrechenden Pyramiden; es schmilat bei $151^{\circ}$ und ist in Alkohol schwer, in Wasser und Äther fast gar nicht löslich.

Das aus m-Diamidobydrazobenzol durch Umlagerung erhaltene $\mathbf{m}$-Diamidobenzidin stimmt mit dem von Täuber (Ber. 23, 797) beschriebenen und aus Dinitrobenzidin erhaltenen überein (Schmelzpunkt $166^{\circ}$ ). 